\title{
AVALIAÇÃO DE LABORATÓRIOS BRASILEIROS NA DETERMINAÇÃO DE ALGUNS PARÂMETROS DE QUALIDADE DE BIOCOMBUSTÍVEIS
}

Mary A. Gonçalves*, Fabiano B. Gonzaga, Isabel C. S. Fraga, Carla M. Ribeiro, Sidney P. Sobral, Eliane C. P. Rego, Elaine B. Santana, Leonardo M. Oliveira, Viviane F. Silva, Rodrigo V. P. Leal, Dalni M. E. Santo Filho, José R. R. Siqueira, Thales P. Barbosa, Janaína M. Rodrigues e Valnei S. Cunha

Instituto Nacional de Metrologia, Qualidade e Tecnologia, Diretoria de Metrologia Científica e Industrial, Av. Nossa Sra. das Graças, 50, 25250-020 Duque de Caxias - RJ, Brasil

Vinícius L. Skrobot, Cristiane B. Costa, Aderson R. Pessoa Júnior, Helena S. P. Carneiro, Helenice Colares, Ednéia Caliman e Márcia V. S. Alves

Agência Nacional de Petróleo, Gás Natural e Biocombustíveis, Superintendência de Biocombustíveis e de Qualidade de Produtos, SGAN 603 módulo H, Asa Norte, 70830-902 Brasília - DF, Brasil

Recebido em 9/5/12; aceito em 26/9/12; publicado na web em 24/1/13

\begin{abstract}
EVALUATION OF BRAZILIAN LABORATORIES FOR DETERMINING QUALITY PARAMETERS OF BIOFUELS. This work shows the results of a Proficiency Testing performed by a partnership between INMETRO and ANP. The performance of 49 Brazilian laboratories (using the $z$-score statistical test) in determining 10 quality parameters of ethanol fuel and biodiesel was evaluated. The certified reference values were provided by INMETRO, allowing a more rigorous assessment of the laboratories. For hydrous ethanol, the acidity parameter showed the lowest number of laboratories with satisfactory results (48\%), while $85 \%$ of the laboratories presented satisfactory results for ethanol content. For biodiesel, the percentage of laboratories with satisfactory results ranged from $46 \%$ (kinematic viscosity) to $92 \%$ (acid number).
\end{abstract}

Keywords: proficiency testing; biofuels quality parameters; certified reference values.

\section{INTRODUÇÃO}

Pioneiro mundial no uso de biocombustíveis, o Brasil alcançou uma posição almejada por muitos países que buscam fontes renováveis de energia, capazes de substituir os derivados de petróleo e contribuir para a redução de emissões de gases de efeito estufa. Os dois principais biocombustíveis líquidos usados no Brasil são o etanol, obtido a partir da cana-de-açúcar e, em escala crescente, o biodiesel, que é produzido a partir de óleos vegetais ou de gorduras animais e adicionado ao diesel de petróleo em proporções variáveis. ${ }^{1}$

O etanol vem sendo utilizado em veículos automotivos desde 1930, época em que esse combustível era misturado à gasolina na proporção de 5\%. Mas, foi através do Programa Brasileiro de Álcool (Proálcool), criado em 1975, que o etanol começou a substituir de forma significativa o uso da gasolina, cujos preços se elevaram subitamente durante uma crise mundial de petróleo. ${ }^{2}$ Atualmente, cerca de $90 \%$ dos veículos comercializados no País são do tipo flex fluel, ${ }^{3}$ que permitem o uso de gasolina e etanol em qualquer proporção. Estudos recentes baseados na avaliação do ciclo de vida desse combustível, que considera os insumos, produtos e impactos envolvidos no processo de produção, transporte e comercialização, demonstraram que a redução de gases de efeito estufa chega a ser maior que $80 \%$ quando comparada à dos combustíveis fósseis. ${ }^{4,5} \mathrm{O}$ Brasil responde por $40 \%$ da oferta mundial de etanol combustível com uma produção anual de aproximadamente 22 trilhões de $\mathrm{L}^{6}$ (safra 2011-2012) e um dos menores custos de produção do mundo (US\$ 0,37/L). ${ }^{5}$

O biodiesel foi introduzido na matriz energética brasileira em 2005 e, atualmente, é obrigatória a adição de 5\% desse biocombustível ao diesel de petróleo comercializado em postos sujeitos à inspeção da Agência Nacional do Petróleo, Gás Natural e Biocombustíveis (ANP), responsável pela regulamentação e

*e-mail: maryinmetro@gmail.com fiscalização do setor de combustíveis no País. O Brasil está entre os maiores produtores e consumidores de biodiesel do mundo, com uma produção anual, em 2011, de 2,6 bilhões de L. ${ }^{1}$ Do total de biodiesel produzido em 2011 , cerca de $70 \%$ foi obtido a partir de óleo de soja, $20 \%$ de gordura bovina e o restante de matrizes diversificadas como óleo de algodão, óleo de palma e óleo de fritura. A produção e o uso do biodiesel no Brasil propiciam o desenvolvimento de uma fonte energética sustentável sob os aspectos ambiental, econômico e social e também trazem a perspectiva da redução das importações de óleo diesel, gerando divisas para o País. O biodiesel apresenta várias vantagens em relação ao diesel de petróleo, pois é um combustível biodegradável e obtido de fontes renováveis. Além disso, durante a sua combustão emite baixos níveis de monóxido de carbono e material particulado, não libera compostos de enxofre e possui melhores propriedades combustíveis, como o número de cetano e a capacidade de lubrificação. ${ }^{7}$

No Brasil existem mais de 400 usinas produtoras de etanol e aproximadamente 50 usinas de biodiesel que têm participado dos leilões da ANP. Aliado a esse fato, há uma grande diversidade de óleos vegetais e gorduras animais que podem ser utilizados na produção de biodiesel, além de diferentes rotas e tipos de catalisadores empregados nas reações de transesterificação. Em função disso, para garantir a comercialização de etanol e biodiesel, é de extrema importância determinar e estipular limites para contaminantes presentes, ou seja, prezar pelo controle de qualidade do produto final. Estudos indicam que a presença de contaminantes está diretamente relacionada com o desempenho dos motores dos veículos, além dos possíveis problemas apresentados pelo uso de combustível de baixa qualidade, como formação de depósitos nas peças metálicas, e entupimento de filtros, bicos injetores e bomba de combustível. ${ }^{8}$ No Brasil, a ANP tem estabelecido em resoluções as especificações de alguns parâmetros físico-químicos de etanol combustível ${ }^{9}$ e biodiesel $^{10}$ utilizados para a avaliação da qualidade desses biocombustíveis. 
Diante desse cenário de valorização dos biocombustíveis e da contínua necessidade de comercializar produtos com qualidade comprovada, o presente trabalho apresenta os resultados de um ensaio de proficiência (EP) nacional realizado através de uma parceria entre a ANP e o Instituto Nacional de Metrologia, Qualidade e Tecnologia (INMETRO). Os parâmetros avaliados tiveram seus valores de referência certificados pelo INMETRO, que por meio de uma cadeia de rastreabilidade metrológica alcançada pelo uso de sistemas primários de medição, técnicas analíticas de alta precisão e exatidão, materiais de referência certificados (MRC) e equipamentos calibrados, é capaz de fornecer resultados confiáveis e metrologicamente comparáveis. ${ }^{11,12}$

Os MRC são utilizados para prover rastreabilidade, confiabilidade e comparabilidade metrológica das medições, para fins de validação de métodos, calibração de equipamentos, além do controle de qualidade nos laboratórios. ${ }^{13}$ Desde 2005, o INMETRO tem estabelecido programas colaborativos com outros Institutos Nacionais de Metrologia e vem atuando de forma pioneira no desenvolvimento de MRC de etanol combustíve ${ }^{12-15}$ e de biodiesel $1{ }^{16}$ pois há uma carência desse tipo de material no mercado nacional e internacional. Entretanto, como há uma grande variedade de biocombustíveis no mercado, esforços têm sido feitos para viabilizar a certificação de todos os parâmetros de qualidade estipulados por organismos de regulação, como a ANP, e também para desenvolver e validar metodologias capazes de prover rastreabilidade metrológica aos resultados dessas medições.

Dessa forma, este EP representa uma oportunidade de se avaliar com mais rigor a proficiência de laboratórios brasileiros na determinação de alguns parâmetros de qualidade de biocombustíveis, fato alcançado por meio da utilização de valores de referência certificados para o cálculo do desempenho, o que até então era feito por meio de um valor de consenso, ou seja, pela média global dos laboratórios participantes.

Para o etanol hidratado foi avaliado o desempenho dos laboratórios na determinação de condutividade elétrica, acidez total, $\mathrm{pH}$, massa específica e teor de etanol, enquanto o etanol anidro foi utilizado exclusivamente para a avaliação do parâmetro teor de água. A condutividade elétrica, a acidez total e o pH têm sido utilizados como indicativos do risco de corrosão que pode ser provocada por etanol de baixa qualidade, e estão relacionados à presença de ácidos e outros contaminantes provenientes dos processos de fermentação e destilação. ${ }^{14,17}$ A massa específica é um dos parâmetros mais utilizados para o controle de qualidade de etanol hidratado, uma vez que está diretamente relacionada ao teor de água contido no produto. $\mathrm{O}$ teor de etanol também é um parâmetro importante para a avaliação da qualidade, uma vez que está relacionado com a pureza do etanol combustível e a possíveis contaminações com água, hidrocarbonetos, metanol e alcoóis superiores. Atualmente, qualquer tipo de gasolina comercializada no Brasil sofre a adição de etanol anidro, cujo percentual atende à legislação vigente (aproximadamente $20-25 \%$ volume). Neste caso, a determinação do teor de água é fundamental para a avaliação da qualidade, pois a solubilidade do etanol na gasolina é inversamente proporcional ao teor de água contido nesse combustível.

Para o biodiesel, os parâmetros avaliados foram índice de acidez, índice de iodo, viscosidade cinemática e massa específica. O índice de acidez é utilizado para estimar o nível de ácidos graxos livres ou produtos de degradação que podem estar presentes nas amostras e, frequentemente, o excesso de ácidos é associado a problemas de corrosão nos motores e degradação de biodiesel durante sua estocagem. ${ }^{18}$ $\mathrm{O}$ índice de iodo permite avaliar o número de insaturações presente no biodiesel, o que está diretamente relacionado com a estabilidade oxidativa, viscosidade e processos de polimerização. ${ }^{19}$ Por outro lado, o controle da viscosidade cinemática é importante para garantir um funcionamento adequado dos sistemas de injeção e bombas de combustível..$^{20} \mathrm{~A}$ massa específica está relacionada com as estruturas moleculares dos alquil ésteres constituintes do biodiesel, sendo utilizada para a avaliação do desempenho desse biocombustível. ${ }^{21}$

Este EP contou com a participação de 49 laboratórios brasileiros e foi realizado de acordo com as diretrizes propostas pela ISO/IEC $17043 .{ }^{22}$ A avaliação estatística dos laboratórios participantes foi realizada por meio do cálculo do índice $z$ (z-score) e para a classificação do desempenho foram utilizados os critérios estabelecidos na ISO $13528 .{ }^{23}$

\section{PARTE EXPERIMENTAL}

\section{Amostras}

Para a realização deste EP foram utilizadas três matrizes diferentes: etanol anidro, etanol hidratado e biodiesel misto obtido a partir de óleo de soja e sebo. O etanol anidro utilizado foi uma amostra de MRC produzido e comercializado pelo INMETRO na forma de ampolas de vidro âmbar de $10 \mathrm{~mL}$, e cada laboratório participante recebeu três ampolas desse material. Para as amostras de etanol hidratado e biodiesel foram utilizados aproximadamente $80 \mathrm{~L}$ de cada biocombustível, que foram acondicionados em garrafas de polietileno de alta densidade de $1 \mathrm{~L}$. Desse total de amostras preparadas, 20 garrafas de cada item a ser utilizado neste EP foram selecionadas aleatoriamente para se realizar a certificação dos parâmetros. Cada laboratório participante recebeu 1 ou 2 garrafas de etanol hidratado e/ou biodiesel, de acordo com o número de parâmetros a ser determinado.

\section{Certificação de etanol combustível e biodiesel}

O INMETRO forneceu os valores de referência certificados e suas respectivas incertezas para os parâmetros de qualidade de etanol e biodiesel por meio dos estudos de homogeneidade, estabilidade e caracterização realizados com base no ISO Guide $35 .{ }^{24} \mathrm{O} \mathrm{MRC}$ de etanol anidro (ampolas de $10 \mathrm{~mL}$ ) também foi desenvolvido com base no ISO Guide 35, sendo utilizado neste EP apenas para a avaliação do parâmetro teor de água.

$\mathrm{O}$ estudo de homogeneidade foi realizado para avaliar se os lotes dos biocombustíveis utilizados possuíam propriedades físico-químicas uniformes. Para cada parâmetro foram analisadas 10 garrafas de etanol hidratado e 10 de biodiesel, em duplicata, em condições de repetibilidade. Para a avaliação estatística dos dados de homogeneidade foi aplicada a análise de variância de fator único (ANOVA), cujos resultados foram posteriormente utilizados para o cálculo de estimativa de incerteza referente à homogeneidade $\left(\mathrm{u}_{\mathrm{hom}}\right)$.

$\mathrm{O}$ estudo de estabilidade foi realizado para verificar o comportamento das amostras durante o período de execução deste EP e foi adotado o modelo clássico, no qual as análises são realizadas em condições de reprodutibilidade. Para isso, as amostras de etanol hidratado foram armazenadas em laboratório com temperatura controlada $\left(20,0 \pm 2,0^{\circ} \mathrm{C}\right)$, enquanto as amostras de biodiesel foram armazenadas em uma câmara fria $\left(4,0 \pm 0,5^{\circ} \mathrm{C}\right)$. Após períodos de tempo pré-estabelecidos (0, 2, 3 e 5 semanas), 2 garrafas de cada item da comparação foram analisadas em triplicata. Os resultados provenientes dessas análises foram avaliados estatisticamente por meio de regressão linear e foram utilizados para o cálculo da estimativa de incerteza referente à estabilidade $\left(\mathrm{u}_{\mathrm{est}}\right)$.

O processo de caracterização provê os valores das propriedades que serão quantificadas. Neste EP adotou-se como valor certificado o valor médio das análises do estudo de homogeneidade. A incerteza da caracterização $\left(\mathrm{u}_{\mathrm{car}}\right)$ foi estimada de acordo com o ISO GUM 25 e o Guia Eurachem/CITAC, ${ }^{26}$ sendo contempladas como fontes de incerteza: a repetibilidade do método (incerteza tipo A), variações das condições ambientais, dados de calibração de equipamentos, preparo 
de amostras e soluções, e outras fontes inerentes à determinação de cada parâmetro (incerteza tipo B). Finalmente, para o cálculo da incerteza padrão combinada $\left(\mathrm{u}_{\text {comb }}\right)$ foi utilizada a Equação 1.

$$
u_{c o m}=\sqrt{\left(u_{h o m}\right)^{2}+\left(u_{e s t}\right)^{2}+\left(u_{c a r}\right)^{2}}
$$

\section{Metodologias}

Os valores certificados foram obtidos com base em métodos normalizados indicados nas Resoluções ANP para etanol combustível ${ }^{9}$ e biodiesel, ${ }^{10}$ ou por meio de metodologias desenvolvidas e validadas no INMETRO, descritas resumidamente a seguir.

\section{Teor de água de etanol anidro}

Determinado por titulação coulométrica tipo Karl Fischer utilizando-se um titulador coulométrico (Metrohm/831), contendo um eletrodo de trabalho duplo de platina (geração de corrente de $400 \mathrm{~mA}$ ) e um eletrodo indicador duplo de platina (detecção amperométrica). A faixa de tensão aplicada na detecção foi de 50 a $70 \mathrm{mV}$. Com o auxílio de uma seringa, uma quantidade de etanol (aproximadamente $0,5 \mathrm{~mL}$ ) foi coletada da ampola e pesada em uma balança analítica (Mettler Toledo/XP205 - resolução 0,01 mg). Em seguida, algumas gotas de etanol (aproximadamente $0,025 \mathrm{~g}$ ) foram injetadas diretamente em uma solução de Hydranal coulomat AG (solução anódica) contendo excesso de iodeto, e a seringa foi pesada novamente para a determinação da massa por diferença. Por meio da passagem de corrente elétrica, o iodeto presente na solução anódica foi oxidado a iodo $\left(\mathrm{I}_{2}\right)$, que posteriormente reagiu com a água presente nas amostras de etanol. O ponto final da titulação foi determinado pelo eletrodo indicador duplo de platina por meio da detecção de excesso de iodo presente no meio. A titulação foi iniciada após a estabilização do equipamento com um drift de $20 \mu \mathrm{g} / \mathrm{min}$ em $120 \mathrm{~s}$ e a curva de titulação foi obtida por meio da medição de pontos a cada $2 \mathrm{~s}$ de análise.

\section{Acidez, total de etanol hidratado}

Determinada por titulação volumétrica com detecção potenciométrica utilizando-se um titulador automático (Titrando Metrohm/836) contendo uma bureta automática de 2,0 mL (resolução $0,0001 \mathrm{~mL}$ ) previamente calibrados pelo INMETRO. Inicialmente, cerca de 40,0 $\mathrm{g}$ de etanol foram pesados em um béquer de $100 \mathrm{~mL}$ utilizando-se uma balança analítica (Mettler Toledo/PR1203 - resolução 0,001 g). Em seguida, a amostra foi titulada com uma solução de $\mathrm{NaOH}$ $(0,0202 \pm 0,0007) \mathrm{mol} / \mathrm{L}$, previamente padronizada com um MRC de $\mathrm{HCl}(0,00881 \pm 0,00008) \mathrm{mol} / \mathrm{kg}$ do INMETRO (código 8134, lote $08.2 / 10.0003$ ). Para a detecção potenciométrica foi utilizado um eletrodo combinado de vidro contendo uma solução etanólica de $\mathrm{LiCl}$ $2,0 \mathrm{~mol} / \mathrm{L}$ como eletrólito interno.

\section{Teor de etanol de etanol hidratado}

Determinado por cromatografia gasosa com detector de ionização de chama (CG-DIC) (cromatógrafo Shimadzu GC/2010) pela técnica de injeção on-column e utilização de 1-propanol para quantificação por padronização interna. Inicialmente, cerca de $0,17 \mathrm{~g}$ de etanol hidratado foram pesados em um frasco de vidro de $5 \mathrm{~mL}$ utilizando-se uma balança analítica (Mettler Toledo/XP205 - resolução 0,01 mg). Em seguida, cada frasco de vidro foi introduzido em outro frasco contendo aproximadamente $250,0 \mathrm{~g}$ de água do tipo I (condutividade eletrolítica igual ou inferior a $0,1 \mu \mathrm{S} / \mathrm{cm}$ ) pesada em uma balança analítica (Sartorius/CPA1003s - resolução 0,001 g) e a solução resultante foi homogeneizada por $12 \mathrm{~h}$. O mesmo procedimento foi realizado para o preparo dos padrões da curva de calibração na faixa de 0,035 a $0,105 \%$ massa e da solução de 1-propanol na concentração de $0,089 \pm 0,001 \%$ massa. Para a realização da análise cromatográfica, cerca de 1,0 g de cada solução (amostras e padrões) foi diluída gravimetricamente na proporção de 1:1 com solução de padrão interno, utilizando-se uma balança analítica (Mettler Toledo/XP205) e, em seguida, cerca de $1,0 \mu \mathrm{L}$ da amostra resultante foi injetada e analisada no cromatógrafo a gás. A determinação do teor de etanol foi realizada pela equação da curva de calibração obtida pelo método dos mínimos quadrados, aplicando-se o modelo linear.

\section{pH de etanol hidratado}

Determinado com base na Norma ABNT NBR 10891, ${ }^{27}$ utilizando-se um medidor de $\mathrm{pH}$ (Metrohm/713) e um eletrodo combinado de vidro contendo uma solução etanólica de $\mathrm{LiCl} 2,0 \mathrm{~mol} / \mathrm{L}$ como eletrólito interno. A calibração do sistema de medição foi realizada a 25,0 $\pm 0,2{ }^{\circ} \mathrm{C}$ utilizando-se MRC de $\mathrm{pH}$ 4,001 \pm 0,026 (código 8832 , lote 03.1/08.0001) e pH 6,857 \pm 0,019 (código 8855, lote 03.2/10.0004), ambos produzidos pelo INMETRO. Os resultados das medições também foram reportados a $25,0 \pm 0,2{ }^{\circ} \mathrm{C}$.

\section{Condutividade elétrica de etanol hidratado}

Determinada com um medidor de condutividade (Metrohm/712) acoplado a uma célula de condutividade não platinizada com constante aproximadamente igual a $0,1 \mathrm{~cm}^{-1}$ (Digimed/DMC-001MS). A calibração do sistema de medição foi realizada a $25,0 \pm 0,2{ }^{\circ} \mathrm{C}$ utilizando-se um MRC de condutividade de 5,06 $\pm 0,10 \mu \mathrm{S} / \mathrm{cm}$ do INMETRO (código 8435, lote 04.5/10.0002) e os resultados das medições foram reportados a essa temperatura.

\section{Massa específica de etanol hidratado e biodiesel}

Determinadas com base nas Normas ISO 15212-1 e ISO $15212-2,{ }^{28}$ utilizando-se para as medições um densímetro digital (DMA/5000) rastreado pelo sistema de pesagem hidrostática (sistema primário de massa específica do Brasil), cujo padrão de referência é uma esfera de silício de $1 \mathrm{~kg}$ calibrada pelo Instituto Nacional de Metrologia do Japão. Esse sistema foi empregado na determinação da massa específica dos materiais de referência utilizados para a calibração do densímetro digital. A calibração foi realizada a 20,00 $\pm 0,02$ ${ }^{\circ} \mathrm{C}$ e os resultados das medições foram reportados a essa temperatura.

\section{Viscosidade cinemática de biodiesel}

Determinada com base nas Normas ISO $3105^{28}$ e ASTM D445, ${ }^{29}$ utilizando-se para as medições um viscosímetro capilar (Ubbelohde/ tipo I) rastreado pela viscosidade da água, de acordo com a norma ISO TR $3666 . .^{28}$ Os resultados das medições foram reportados a $40,00 \pm 0,02{ }^{\circ} \mathrm{C}$.

\section{Índice de acidez de biodiesel}

Determinado com base na Norma ASTM D664. ${ }^{29}$ Para isso foi realizada titulação volumétrica com detecção potenciométrica utilizando-se um titulador automático (Titrino Metrohm/785-DMP) contendo uma bureta automática de $20,0 \mathrm{~mL}$ (resolução $0,001 \mathrm{~mL}$ ) previamente calibrados pelo INMETRO. Inicialmente, cerca de 5,0 $\mathrm{g}$ de biodiesel foram pesados em um béquer de $100 \mathrm{~mL}$ utilizando-se uma balança analítica (Mettler Toledo/AG285 - resolução 0,01 mg). Em seguida, a amostra foi diluída em $50 \mathrm{~mL}$ de isopropanol (pureza $99,9 \%$ ) e titulada com uma solução de $\mathrm{KOH}$ em isopropanol 0,0124 $\pm 0,0005 \mathrm{~mol} / \mathrm{L}$, previamente padronizada com um MRC de KHP de pureza 99,809 \pm 0,014\% produzido pelo INMETRO (código 8118, lote 12.2/09.0001). Para a detecção potenciométrica foi utilizado um eletrodo combinado de vidro contendo uma solução etanólica de $\mathrm{LiCl}$ 2,0 $\mathrm{mol} / \mathrm{L}$ como eletrólito interno.

Índice de iodo de biodiesel

Determinado com base na Norma EN $14111 .{ }^{30}$ Para isso foi 
realizada titulação volumétrica com detecção potenciométrica utilizando-se um titulador automático (Titrando Metrohm/857) contendo uma bureta automática de 20,0 $\mathrm{mL}$ (resolução $0,001 \mathrm{~mL}$ ) previamente calibrados pelo INMETRO. Para realizar a titulação cerca de 0,15 $\mathrm{g}$ de biodiesel foram pesados utilizando-se uma balança analítica (Sartorius/ME235S - resolução 0,01 mg) e, em seguida, a amostra foi dissolvida em $20 \mathrm{~mL}$ de solvente composto por cicloexano e ácido acético na proporção de 1:1. A essa solução foram acrescentados 25 $\mathrm{mL}$ de solução de iodo de Wijs e a mistura resultante permaneceu em ambiente fechado e escuro por $1 \mathrm{~h}$. Posteriormente foram adicionados à mistura $20 \mathrm{~mL}$ de solução de iodeto de potássio na concentração de $10 \%$ massa e $150 \mathrm{~mL}$ de água desionizada. Finalmente essa mistura foi titulada com uma solução de tiossulfato de sódio recém-preparada $0,101 \pm 0,004 \mathrm{~mol} / \mathrm{L}$, previamente padronizada com dicromato de potássio (pureza 99,9\%). Para a detecção potenciométrica foi utilizado um eletrodo fio duplo de platina.

Para a determinação dos parâmetros de qualidade de etanol e biodiesel, os laboratórios participantes utilizaram obrigatoriamente um dos métodos normalizados (ABNT, ASTM ou EN) especificados pela $\mathrm{ANP}^{9,10}$ e descritos na Tabela 1.

\section{Avaliação estatística dos resultados dos laboratórios}

A avaliação estatística dos laboratórios participantes foi realizada por meio do cálculo do índice $z$ utilizando-se a Equação 2.

$$
Z=\frac{x-X}{\hat{\sigma}}
$$

onde: $x=$ resultado do laboratório participante; $X=$ valor de referência fornecido pelo INMETRO, denominado valor designado; $\hat{\sigma}=$ desvio padrão do EP determinado pelo comitê técnico.

\section{RESULTADOS E DISCUSSÃO}

\section{Certificação de etanol combustível e biodiesel}

Os valores certificados e as respectivas incertezas dos parâmetros de etanol e biodiesel avaliados neste EP foram determinados por meio de medições analíticas realizadas nos estudos de homogeneidade, estabilidade e caracterização das amostras. Os dados da análise estatística (ANOVA) indicaram que as contribuições relativas das incertezas da homogeneidade com relação ao valor certificado foram inferiores a $3 \%$. Para a estabilidade, os dados de regressão linear $(p>0,05)$ comprovaram que as amostras se mantiveram estáveis durante todo o período de execução do EP. Os resultados dos valores certificados com suas respectivas incertezas combinadas são mostrados na Tabela 2. Para cada parâmetro avaliado, foram adotados como valor designado e desvio padrão do EP (cálculo do índice $z$ ) o resultado obtido na certificação das amostras e sua incerteza combinada, respectivamente, ambos arredondados para o número de casas decimais que foi estipulado pela ANP no formulário de registro de resultados utilizado pelos laboratórios. A incerteza combinada foi sempre arredondada para cima de forma a não prejudicar a avaliação dos laboratórios participantes. Os valores designados e seus respectivos desvios padrão, bem como os valores limite especificados pela $\mathrm{ANP}^{9,10}$ para cada parâmetro também são dados na Tabela 2.

Como pode ser observado na Tabela 2, todos os parâmetros avaliados neste EP apresentaram valores certificados dentro dos limites especificados nas Resoluções da ANP para etanol e biodiesel.

\section{Avaliação dos laboratórios participantes}

Para todos os parâmetros de etanol e biodiesel avaliados, os resultados dos laboratórios foram avaliados por meio de gráficos de dispersão e do cálculo do índice $z$. A avaliação do desempenho pelo índice $z$ foi realizada com base nos seguintes critérios: $|z| \leq 2$ : Resultado satisfatório; $2<|z| \leq 3$ : Resultado questionável; $|z|>3$ : Resultado insatisfatório.

Como a sistemática de avaliação dos resultados foi a mesma para todos os parâmetros, o pH de etanol hidratado foi adotado, como exemplo, para a discussão dos dados. Na Figura 1 são mostrados os resultados de todos os laboratórios participantes na determinação desse parâmetro, a comparação desses dados com o valor certificado e sua incerteza combinada.

Para uma avaliação mais criteriosa, foram estabelecidos os limites do valor certificado associado a uma, duas ou três vezes o valor da incerteza combinada. Os laboratórios participantes realizaram as medições em triplicata, o que permitiu realizar os cálculos do valor médio e do desvio padrão, ambos representados na Figura 1, sendo que o desvio padrão está apresentado na forma de barra de erros. É importante destacar que o desvio padrão contempla apenas a repetibilidade do método, o que não corresponde à incerteza do parâmetro, cujo valor depende da repetibilidade e de outras fontes como mencionado anteriormente. Dos 37 laboratórios participantes que reportaram resultados de $\mathrm{pH}$ de etanol hidratado, 9 (24,3\%) apresentaram resultados muito próximos do valor certificado $\pm \mathrm{u}_{\text {comb }}$ $(6,28 \pm 0,13) ; 6(16,2 \%)$ apresentaram resultados dentro da faixa do valor certificado $\pm 2 \mathrm{u}_{\text {comb }}(6,28 \pm 0,26) ; 6(16,2 \%)$ apresentaram

Tabela 1. Métodos normalizados utilizados pelos laboratórios participantes para a determinação dos parâmetros de etanol combustível e biodiesel avaliados nesse EP

\begin{tabular}{|c|c|c|c|c|}
\hline \multirow{2}{*}{ Combustível } & \multirow{2}{*}{ Parâmetro } & \multicolumn{3}{|c|}{ Normas } \\
\hline & & ABNT & ASTM & $\mathrm{EN}$ \\
\hline Etanol anidro & Teor de água ${ }^{27}$ & NBR 15888 & - & - \\
\hline \multirow[t]{5}{*}{ Etanol hidratado } & Massa específica a $20{ }^{\circ} \mathrm{C}^{27,29}$ & NBR 14065 & ASTM D 4052 & - \\
\hline & Acidez total ${ }^{27,29}$ & NBR 9866 & ASTM D 1613 & - \\
\hline & Teor de etanol ${ }^{29}$ & - & ASTM D 5501 & - \\
\hline & $\mathrm{pH}^{27}$ & NBR 10891 & - & - \\
\hline & Condutividade elétrica ${ }^{27}$ & NBR 10547 & - & - \\
\hline \multirow[t]{4}{*}{ Biodiesel } & Massa específica a $20^{\circ} \mathrm{C}^{27,29,30}$ & NBR 14065 & ASTM D 4052 & EN ISO 12185 \\
\hline & $\begin{array}{l}\text { Viscosidade cinemática a } 40 \\
{ }^{\circ} \mathrm{C}^{27,29,30}\end{array}$ & NBR 10441 & ASTM D 445 & EN ISO 3104 \\
\hline & Índice de acidez ${ }^{27,29,30}$ & NBR 14448 & ASTM D 664 & EN 14104 \\
\hline & Índice de iodo $\mathrm{o}^{30}$ & - & - & EN 14111 \\
\hline
\end{tabular}


Tabela 2. Valores certificados com suas respectivas incertezas combinadas, valores designados, desvios padrão do EP e especificação ANP para os parâmetros de etanol combustível e biodiesel

\begin{tabular}{|c|c|c|c|c|c|c|}
\hline Combustível & Parâmetro & $\begin{array}{c}\text { Valor certificado } \pm \\
\mathrm{u}_{\text {comb }}\end{array}$ & Valor designado & $\begin{array}{c}\text { Desvio padrão } \\
\text { do EP } \\
\end{array}$ & $\begin{array}{l}\text { Especificação } \\
\text { ANP }\end{array}$ & Unidade \\
\hline Etanol anidro & Teor de água ${ }^{a}$ & $(0,244 \pm 0,004)$ & --- & --- & 0,4 (máx.) & $\%$ volume \\
\hline \multirow[t]{5}{*}{ Etanol hidratado } & $\begin{array}{c}\text { Massa específica a } \\
20{ }^{\circ} \mathrm{C}\end{array}$ & $(810,14 \pm 0,05)$ & 810,1 & 0,1 & 807,6 a 811,0 & $\mathrm{~kg} / \mathrm{m}^{3}$ \\
\hline & Acidez total & $(8,52 \pm 0,42)$ & 8,5 & 0,5 & 30 (máx.) & $\mathrm{mg} / \mathrm{L}$ \\
\hline & Teor de etanol & $(95,0 \pm 0,7)$ & 95,0 & 0,7 & 94,5 (mín.) & $\%$ volume \\
\hline & $\mathrm{pH}$ & $(6,28 \pm 0,13)$ & 6,3 & 0,2 & 6,0 a 8,0 & --- \\
\hline & $\begin{array}{l}\text { Condutividade } \\
\text { elétrica }\end{array}$ & $(187 \pm 15)$ & 187 & 15 & 350 (máx.) & $\mu \mathrm{S} / \mathrm{m}$ \\
\hline \multirow[t]{4}{*}{ Biodiesel } & $\begin{array}{c}\text { Massa específica a } \\
20^{\circ} \mathrm{C}\end{array}$ & $(881,35 \pm 0,04)$ & 881,4 & 0,1 & 850 a 900 & $\mathrm{~kg} / \mathrm{m}^{3}$ \\
\hline & $\begin{array}{l}\text { Viscosidade cin- } \\
\text { emática a } 40{ }^{\circ} \mathrm{C}\end{array}$ & $(4,1394 \pm 0,0048)$ & 4,139 & 0,005 & 3,0 a 6,0 & $\mathrm{~mm}^{2} / \mathrm{s}$ \\
\hline & Índice de acidez & $(0,368 \pm 0,045)$ & 0,37 & 0,05 & 0,5 (máx.) & $\mathrm{mg} \mathrm{KOH} / \mathrm{g}$ \\
\hline & Índice de iodo & $(127,9 \pm 3,7)$ & 128 & 4 & Anotar & $\mathrm{g} \mathrm{I}_{2} / 100 \mathrm{~g}$ \\
\hline
\end{tabular}

aNão foi utilizado o índice $z$, os laboratórios foram avaliados considerando-se o valor certificado e sua incerteza expandida para um nível de confiança de $99 \%$ $\left(\mathrm{u}_{\text {comb }} \times 3\right)$

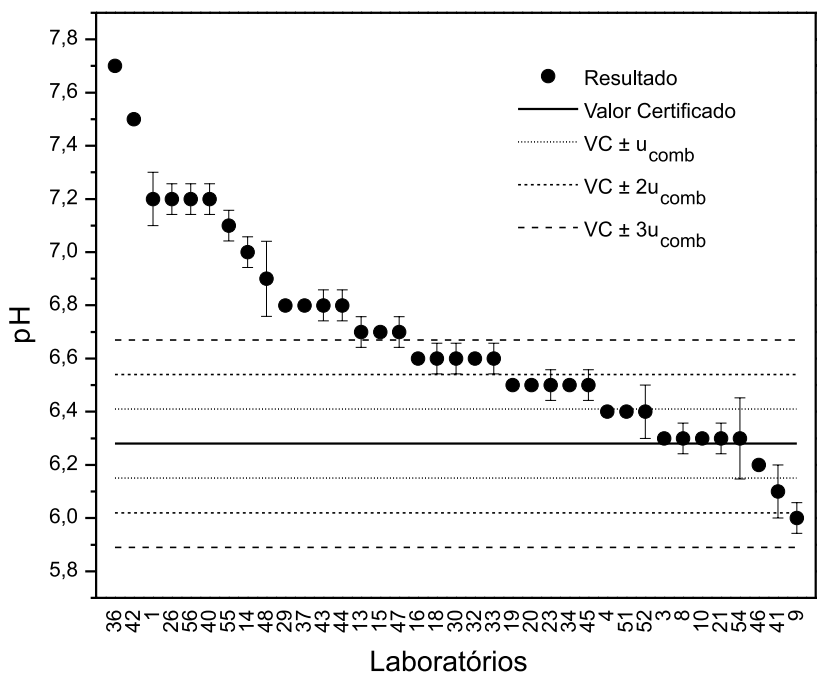

Figura 1. Resultados dos laboratórios participantes para o parâmetro $\mathrm{pH}$ de etanol hidratado e comparação com o valor certificado. O desvio padrão dos resultados de cada laboratório está representado na forma de barra de erros

resultados dentro da faixa do valor certificado $\pm 3 \mathrm{u}_{\text {comb }}(6,28 \pm 0,39)$ e $16(43,3 \%)$ informaram um valor de $\mathrm{pH}$ maior que o valor certificado $\pm 3 \mathrm{u}_{\text {comb }}(>6,67)$. Na Figura 2 são apresentados os dados do índice $z$ para o parâmetro $\mathrm{pH}$ de etanol hidratado.

A partir da Figura 2 é possível verificar que, dos 37 laboratórios participantes, $24(64,9 \%)$ apresentaram resultados satisfatórios $(|z|$ $\leq 2) ; 5(13,5 \%)$ resultados questionáveis $(2<|z| \leq 3)$ e $8(21,6 \%)$ resultados insatisfatórios $(|z|>3)$. Comparando-se as Figuras 1 e 2, pode ser observado que todos os laboratórios cujos resultados foram insatisfatórios $(|z|>3)$ e questionáveis $(2<|z| \leq 3)$ apresentaram valores de $\mathrm{pH}$ maiores que o valor certificado $\pm 3 \mathrm{u}_{\text {comb }}$ (laboratórios 36 , 42, 1, 26, 56, 40, 55, 14, 48, 29, 37, 43 e 44). Os laboratórios 1, 48, 52,54 e 41 apresentaram desvios padrão mais elevados que o restante do grupo, sendo que os laboratórios 1 e 48 apresentaram resultados insatisfatório e questionável, respectivamente. Em contrapartida, os laboratórios 52, 54 e 41 apresentaram resultados satisfatórios (índice $z$ igual a 0,$5 ; 0,0$ e $-1,0$; respectivamente). Isso significa que esses

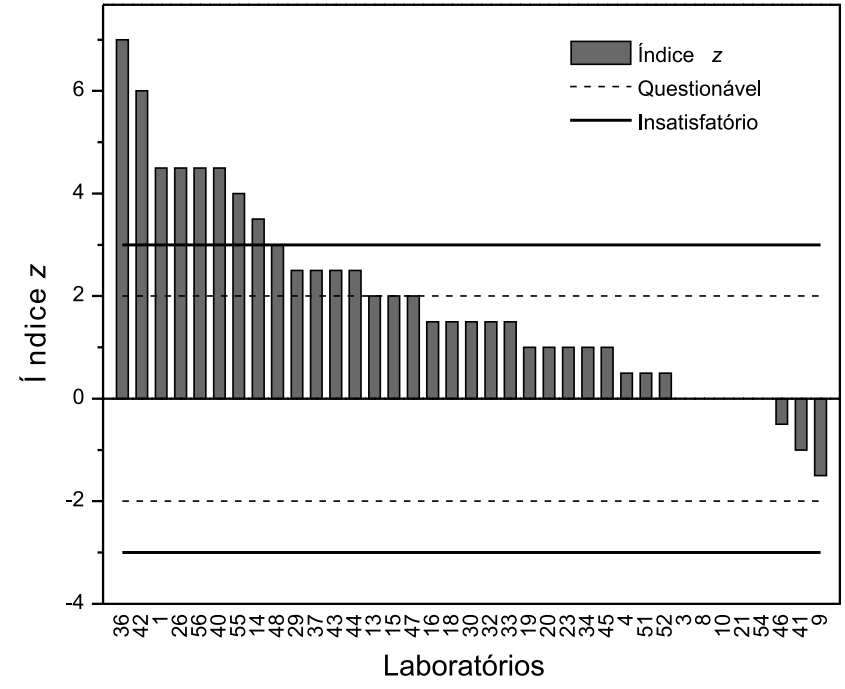

Figura 2. Índice z dos laboratórios para o parâmetro $\mathrm{pH}$ de etanol hidratado

três laboratórios forneceram resultados médios próximos ao valor de referência (valor certificado $\pm \mathrm{u}_{\text {comb }}$ ), ou seja, exatidão satisfatória, mas as replicatas das medições apresentaram uma variabilidade considerável, indicando que a repetibilidade (precisão) do método desses laboratórios precisa ser melhorada.

Para realizar uma avaliação mais objetiva, o desempenho dos laboratórios participantes foi calculado em percentual de laboratórios com resultados satisfatórios, questionáveis e insatisfatórios. Os resultados para os parâmetros massa específica, acidez total, teor de etanol, $\mathrm{pH}$ e condutividade elétrica de etanol hidratado são mostrados na Figura 3.

Na Figura 3 pode-se observar que para o etanol hidratado, o maior percentual de laboratórios com resultados satisfatórios (85\%) foi obtido na determinação do teor de etanol, fato que evidencia a equivalência entre a metodologia utilizada pelos laboratórios (ASTM D5501 ${ }^{29}$ ) e o método empregado pelo INMETRO para a determinação do valor de referência. Neste EP, a acidez total apresentou o menor percentual de laboratórios com resultados satisfatórios (48\%), o que pode estar associado a questões como preparo de reagentes e ao fato 


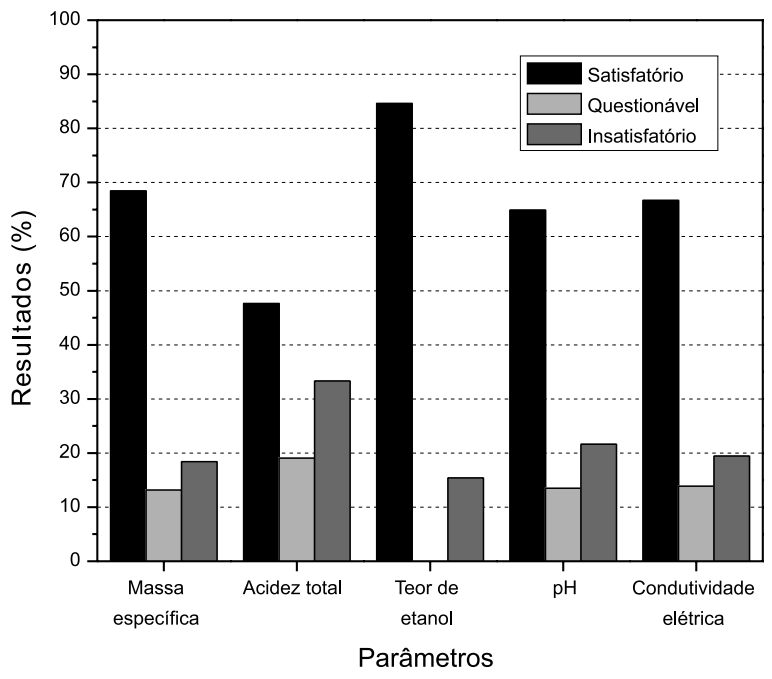

Figura 3. Percentual de laboratórios com resultados satisfatórios, questionáveis e insatisfatórios dentre os participantes avaliados nesse EP para a análise de etanol hidratado

da técnica ser muito dependente do discernimento prático do analista (velocidade de titulação, detecção do ponto final da titulação e agitação da amostra). Para a obtenção do valor certificado de acidez total, o INMETRO utilizou uma metodologia baseada em titulação potenciométrica, cuja validação foi realizada por meio de uma comparação interlaboratorial. ${ }^{31}$ Nesse estudo ficou comprovado estatisticamente que o método de titulação potenciométrica é equivalente ao método descrito na Norma ABNT NBR 9866 para amostras de etanol com acidez total variando de 5 a $50 \mathrm{mg} / \mathrm{L}$. Para $\mathrm{pH}$, condutividade elétrica e massa específica a $20^{\circ} \mathrm{C}$, o percentual de laboratórios com resultados satisfatórios foi igual a 65,67 e $68 \%$, respectivamente.

Para o parâmetro teor de água de etanol anidro, a avaliação do desempenho dos laboratórios em relação ao valor de referência foi diferenciada. Considerando-se que os laboratórios reportaram os resultados com uma casa decimal, e a incerteza combinada do valor de referência $(0,004 \%$ volume) foi obtida com uma precisão muito superior à resolução dos resultados dos laboratórios ( $0,1 \%$ volume), a utilização do índice $z$ tornou-se impraticável. Assim, para a avaliação desses resultados, foi considerado como referência o valor certificado e sua incerteza expandida para um nível de confiança de $99 \%$, obtida multiplicando-se a incerteza combinada pelo fator de abrangência três $(k=3)$. Nesse caso, o desempenho dos laboratórios foi avaliado utilizando-se o valor $0,244 \pm 0,012 \%$ volume com o arredondamento dos limites inferior e superior para uma casa decimal, sendo considerados satisfatórios os resultados dentro da faixa de 0,2 a $0,3 \%$ volume. Com base neste critério, dos 15 laboratórios que realizaram o ensaio de teor de água, 14 (93\%) obtiveram resultados considerados satisfatórios, o que comprova a equivalência entre os resultados dos laboratórios participantes e o valor de referência certificado pelo INMETRO.

O percentual de laboratórios com resultados satisfatórios, questionáveis e insatisfatórios para os parâmetros de biodiesel avaliados são mostrados na Figura 4.

Para o biodiesel, o índice de acidez apresentou o maior percentual de laboratórios com resultados satisfatórios, 92\%, indicando que as metodologias utilizadas pelos laboratórios participantes e pelo INMETRO apresentaram boa concordância. Para a massa específica a $20{ }^{\circ} \mathrm{C}, 90 \%$ dos laboratórios apresentaram resultados considerados satisfatórios e para o índice de iodo, $81 \%$. Neste EP apenas $46 \%$ dos laboratórios apresentaram resultados satisfatórios na determinação da viscosidade cinemática a $40{ }^{\circ} \mathrm{C}$. Para esse último parâmetro,

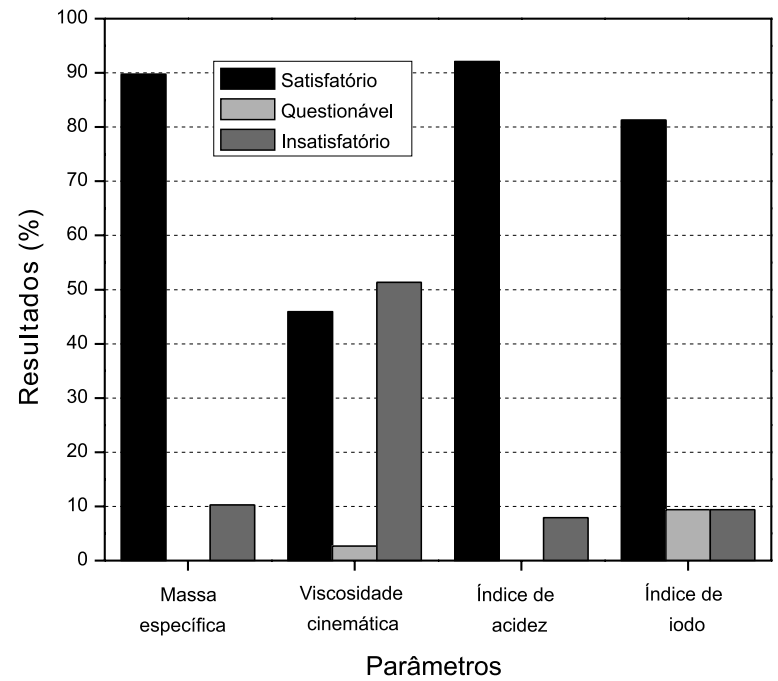

Figura 4. Percentual de laboratórios com resultados satisfatórios, questionáveis e insatisfatórios dentre os participantes avaliados nesse EP para a análise de biodiesel

isso pode indicar a necessidade de validação da metodologia para biodiesel, já que os métodos disponíveis foram elaborados para produtos de petróleo.

\section{CONCLUSÃO}

Este EP foi realizado por meio da cooperação técnica estabelecida entre o INMETRO e a ANP, permitindo a avaliação da proficiência de 49 laboratórios brasileiros na determinação de 10 parâmetros de qualidade de etanol combustível e biodiesel utilizando-se amostras com valores de referência certificados. O desempenho dos laboratórios foi avaliado pelo cálculo do índice $z$, tendo sido observado, de maneira geral, um maior percentual de resultados satisfatórios. Para o etanol hidratado, os melhores resultados foram alcançados na determinação do parâmetro teor de etanol, para o qual $85 \%$ dos laboratórios apresentaram resultados satisfatórios. Por outro lado, para a acidez total apenas $48 \%$ dos laboratórios participantes apresentaram $|z| \leq 2$ (desempenho satisfatório). Para o biodiesel, o percentual de laboratórios com resultados satisfatórios variou de $46 \%$ (viscosidade cinemática) a $92 \%$ (índice de acidez).

Os valores de referência certificados e as respectivas incertezas dos parâmetros de etanol combustível e biodiesel foram obtidos por meio dos estudos de homogeneidade, estabilidade e caracterização das amostras. Esse processo de certificação foi realizado pelo INMETRO e contribuiu de forma significativa para o aumento da confiabilidade e rastreabilidade metrológica das medições e para a realização de uma análise estatística mais criteriosa. Dessa forma, por meio deste EP foi possível realizar uma avaliação mais rigorosa e restritiva dos laboratórios, uma vez que a proficiência foi avaliada com base em um valor de referência e não por um valor de consenso, cujo resultado poderia ser afetado pelo desempenho global dos participantes. Em contrapartida, os laboratórios poderão identificar possíveis problemas em seus procedimentos de medição e implementar ações corretivas e de melhoria, demonstrando que um EP também constitui uma ferramenta de educação e autoaperfeiçoamento.

\section{REFERÊNCIAS}

1. http://www.anp.gov.br, acessada em Janeiro 2013.

2. Moreira, J. R.; Goldemberg, J.; Energy Policy 1999, 27, 229; Mussatto, S. I.; Dragone, G.; Guimarães, P. M. R.; Silva, J. P. A.; Carneiro, L. M.; 
Roberto, I. C.; Vicente, A.; Domingues, L.; Teixeira, J. A.; Biotechnol. Adv. 2010, 28, 817.

3. Leite, R. C. C.; Leal, M. R. L. V.; Cortez, L. A. B.; Griffin, W. M.; Scandiffio, M. I. G.; Energy 2009, 34, 655.

4. Goldemberg, J.; Coelho, S. T.; Guardabassi, P.; Energy Policy 2008, 36, 2086; Macedo, I. C.; Seabra, J. E. A.; Silva, J. E. A. R.; Biomass Bioenergy 2008, 32, 582.

5. Lora, E. E. S.; Palacio, J. C. E.; Rocha, M. H.; Renó, M. L. G.; Venturini, O. J.; Olmo, O. A. D.; Energy 2011, 36, 2097.

6. http://mapas.agricultura.gov.br:81/Spc/daa/Resumos/acompanhamento_ producao_16_01_2012_11-12.pdf, acessada em Janeiro 2013.

7. Zhang, Y.; Dubé, M. A.; McLean, D. D.; Kates, M.; Bioresour. Technol. 2003, 89, 1; Pousa, G. P. A. G.; Santos, A. L. F.; Suarez, P. A. Z.; Energy Policy 2007, 35, 5393; Cordeiro, C. S.; Silva, F. R.; Wypych, F.; Ramos, L. P.; Quim. Nova 2011, 34, 477; Iriarte, A.; Rieradevall, J.; Gabarrell, X.; Appl. Energy. 2012, 91, 263.

8. Knothe, G.; Trans. ASAE 2001, 44, 193; Agarwal, A. K.; Prog. Energy Combust. Sci. 2007, 33, 233; Wang, H.; Tang, H.; Wilson, J.; Salley, O. S.; Simon Ng, K. Y.; J. Am. Oil Chem. Soc. 2008, 85, 1083; Oliveira, M. F.; Saczk, A. A.; Okumura, L. L.; Stradiotto, N. R.; Energy Fuels 2009, 23, 4852.

9. Resolução ANP N 7 (DOU 10.2.2011), http://www.anp.gov.br, acessada em Janeiro 2013.

10. Resolução ANP N ${ }^{\circ} 7$ (DOU 20.3.2008), http://www.anp.gov.br, acessada em Janeiro 2013.

11. Bergoglio, M.; Malengo, A.; Mari, D.; Measurement 2011, 44, 1461.

12. Cortez, L. A. B.; Sugarcane Bioethanol, Blucher: São Paulo, 2010, part 4, cap. 21.

13. Rodrigues, J. M.; Guimarães, E. F.; Sousa, M. V. B.; Silva, V. F.; Fraga, I. C. S.; Souza, V.; Cunha, V. S.; Quim. Nova 2012, 35, 1011.

14. Fraga, I. C. S.; Ribeiro, C. M.; Sobral, S. P.; Dias, J. C.; Gonçalves, M. A.; Borges, P. P.; Gonzaga, F. B.; Talanta 2012, 99, 99.

15. http://www.inmetro.gov.br/metcientifica/mat_ref_cert.asp, acessada em Janeiro 2013.

16. http://www.nist.gov/srm/, acessada em Janeiro 2013.

17. Gonzaga, F. B.; Gonçalves, M. A.; Sobral, S. P.; Ribeiro, C. M.; Fuel 2012, 94, 70; Gonçalves, M. A.; Gonzaga, F. B.; Fraga, I. C. S.; Ribeiro, C. M.; Sobral, S. P.; Borges, P. P.; Rocha, W. F. C.; Sens. Actuators, B 2011, 158, 327.

18. Gonçalves, M. A.; Cunha, K. C.; Sobral, S. P.; Gonzaga, F. B.; Fraga, I. C. S.; Borges, P. P.; J. ASTM Int. 2011, 8, No 2; Gonzaga, F. B.; Sobral, S. P.; Talanta 2012, 97, 199.

19. Leal, R. V. P.; Borges, P. P.; Seidl, P. R.; J. ASTM Int. 2011, 8, № 2.

20. Hoekman, S. K.; Broch, A.; Robbins, C.; Ceniceros, E.; Natarajan, M.; Renewable Sustainable Energy Rev. 2012, 16, 143.

21. Lôbo, I. P.; Ferreira, S. L. C.; Cruz, R. S.; Quim. Nova 2009, 32, 1596.

22. International Organization for Standardization (ISO); ISO/IEC 17043:2010, Conformity assessment - General requirements for proficiency testing, Geneva, 2010, http://www.iso.org/iso/iso_catalogue, acessada em Janeiro 2013.

23. International Organization for Standardization (ISO); ISO 13528:2005,
Statistical methods for use in proficiency testing by interlaboratory comparisons, Geneva, 2005, http://www.iso.org/iso/iso_catalogue, acessada em Janeiro 2013.

24. International Organization for Standardization (ISO); ISO Guide 35:2006, Reference Materials - General and Statistical Principles for Certification, Geneva, 2006, http://www.iso.org/iso/iso_catalogue, acessada em Janeiro 2013.

25. Associação Brasileira de Norma Técnicas (ABNT); Guia para a Expressão da Incerteza de Medição, $3^{\mathrm{a}}$ ed. brasileira do Guide to the Expression of Uncertainty in Measurement - ISO GUM; ABNT: Rio de Janeiro, 2003

26. EURACHEM; Quantifying Uncertainty in Analytical Measurement, $2^{\text {nd }}$ ed., EURACHEM: Helsinki, 2000.

27. Associação Brasileira de Norma Técnicas (ABNT); ABNT NBR 10891:2006, Álcool etílico hidratado - Determinação do $\mathrm{pH}$ - Método potenciométrico; ABNT NBR 15888:2010, Etanol - Determinação do teor de água - Método coulométrico de Karl Fischer; ABNT NBR 9866:2010, Álcool etílico - Determinação da acidez total; ABNT NBR 10547:2006, Álcool etílico - Determinação da condutividade elétrica; ABNT NBR 14448:2009, Produtos de petróleo - Determinação do número de acidez pelo método de titulação potenciométrica, http://www. abntcatalogo.com.br, acessada em Janeiro 2013.

28. International Organization for Standardization (ISO); ISO 152121:1998, Oscillation-type density meters - Part 1: Laboratory Instruments; ISO 15212-2:2002, Oscillation-type density meters - Part 2: Process instruments for homogeneous liquids; ISO 3105:1994, Glass capillary kinematic viscometers - Specifications and operating instructions; ISO TR 3666:1998, Viscosity of water, http://www.iso.org/iso/ iso_catalogue, acessada em Janeiro 2013.

29. ASTM International; ASTM D445:2011, Standard Test Method for Kinematic Viscosity of Transparent and Opaque Liquids (and Calculation of Dynamic Viscosity); ASTM D664:2011, Standard Test Method for Acid Number of Petroleum Products by Potentiometric Titration; ASTM D1613:2006, Standard Test Method for Acidity in Volatile Solvents and Chemical Intermediates Used in Paint, Varnish, Lacquer, and Related Products; ASTM D5501:2009, Standard Test Method for Determination of Ethanol Content of Denatured Fuel Ethanol by Gas Chromatography, http://www.astm.org/Standard/index.shtml, acessada em Janeiro 2013.

30. European Committee for Standardization; EN 14111:2003, Fat and oil derivatives - Fatty Acid Methyl Esters (FAME) - Determination of iodine value; EN ISO 12185:1996, Crude petroleum and petroleum products - Determination of density - Oscillating U-tube method; EN ISO 3104:1996, Petroleum products - Transparent and opaque liquids - Determination of kinematic viscosity and calculation of dynamic viscosity; EN 14104:2003, Fat and oil derivatives - Fatty Acid Methyl Esters (FAME) - Determination of acid value; EN 14111:2003, Fat and oil derivatives - Fatty Acid Methyl Esters (FAME) - Determination of iodine value, http://www.cen.eu/cen/pages/default.aspx, acessada em Janeiro 2013.

31. http://repositorios.inmetro.gov.br/handle/10926/1193, acessada em Janeiro 2013. 